\title{
Octahedral rotation-induced ferroelectricity in cation ordered perovskites
}

\author{
James M. Rondinelli, ${ }^{1, *}$ and Craig J. Fennie ${ }^{2}$, \\ ${ }^{1}$ Department of Materials Science \& Engineering, Drexel University, Philadelphia, PA 19104, USA \\ ${ }^{2}$ School of Applied 85 Engineering Physics, Cornell University, Ithaca, New York 14853, USA
}

(Dated: October 22, 2018)

Increasing demands for electric field-tunable electric, magnetic, and orbital (EMO) materials has renewed interests in ferroelectricity and its coupling to EMO properties. Materials where a spontaneous electric polarization $P$, which naturally couples to and is switched by an electric field, strongly interacts with EMO degrees of freedom provide a platform to realize novel electric-field-controllable, low-power multifunctional devices such as ultra-fast Mottbased devices ${ }^{1] 8}$ In the versatile class of complex $A B \mathrm{O}_{3}$ perovskite oxides (Fig. 1), ferroelectric polarizations are usually induced by polar displacements of second-order Jahn-Teller (SOJT) active cations $\$ 11$ on the $B$-site (commonly $d^{0}$ transition metals ions) and/or on the $A$-site (lone-pair active cations). The cation displacements that induce ferroelectricity, however, are often incompatible with and/or weakly coupled $\frac{12 \mid 13}{1}$ to EMO-derived material properties such as electronic bandwidths, $\frac{14 \mid 15}{1-15}$ magnetic interactions $\frac{16 \mid 17}{}$ and critical transition temperatures. $\frac{18 \mid 19}{19}$ Rotations of corner-sharing $B \mathrm{O}_{6}$ octahedra, however, directly alter these macroscopic properties, $\stackrel{20}{, 2}$ because they buckle the inter-octahedral $B-\mathrm{O}-B$ bond angles which mediate the interplay of EMO degrees of freedom. Thus in perovskites, although the vast number of chemical compositions available (Fig. 1 1 ) facilitates nearly every conceivable material property, the local polar cation displacements necessary for ferroelectricity often occur independently of the property-controlling and pervasive $21+23$ $\mathrm{BO}_{6}$ rotations in the extended framework-rotations account for nearly $75 \%$ of all rhombohedral, Fig. 1 b, and orthorhombic, Fig. 1; , structures.

The strong coupling of oxygen octahedral rotations to the EMO properties in the perovskite structure presents an opportunity to create novel multifunctional materials that respond to external electric fields. The steric linkage of the $\mathrm{BO}_{6}$ framework, however, constrains rotations of octahedra in the same plane to have equal magnitudes but opposite rotational "sense," thereby preserving inversion symmetry ${ }^{[25}$ Thus collective rotation patterns do not induce an electric polarization nor directly couple to an electric field. To circumvent this reality, synthetic chemical routes have focused on turning the centric $\mathrm{BO}_{6}$ octahedra into acentric structural units by partial halide substitution. ${ }^{26} 28$ Progress in cis- and trans-ordering of the halide anions throughout all acentric units, however, remains slow and ferroelectric switching even more challenging. ${ }^{291}$ Recently, a new strategy involving the layering of perovskites blocks $\sqrt{32} \sqrt[35]{3}$ has lead to the realization of ferroelectric systems whose electric polarizations can be completely accounted for by centric rotations. The mechanism has been referred to as hybrid

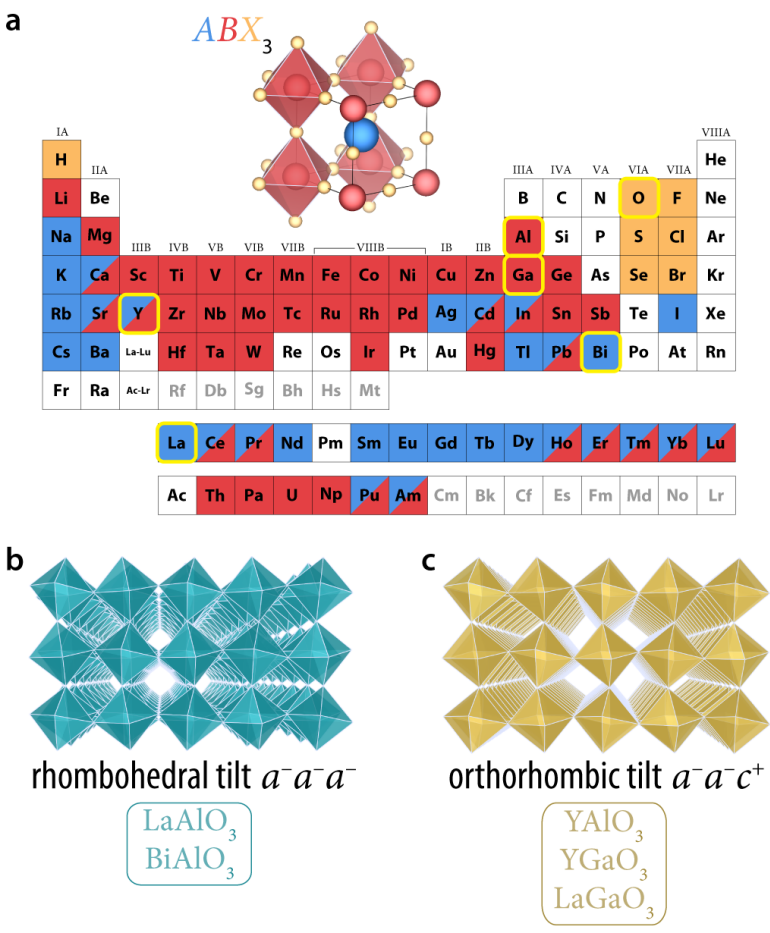

FIG. 1. The ubiquitous octahedral rotations available to perovskites regardless of chemistry preserve inversion symmetry. (a) The adaptable $A B X_{3}$ perovskite structure permits a vast number of chemical compositions, indicated by the colorcoded periodic table, and therefore also exhibits nearly every conceivable materials property. The oxide material class, especially the compositions studied here (listed below), exhibits a network of corner-connected $\mathrm{BO}_{6}$ octahedra which are often distorted from the cubic structure shown in (a). The pervasive low symmetry (centrosymmetric) rhombohedral (b) and orthorhombic (c) structures that result are due to rotations of the $\mathrm{BO}_{6}$ building blocks. Following Glazer notation, $\frac{21}{2}$ the $a^{-} a^{-} a^{-}$octahedral tilt pattern in (b) consists of rotations of adjacent $\mathrm{BO}_{6}$ octahedra that are out-of-phase (-) and of equal magnitude along all Cartersian directions. The $a^{-} a^{-} c^{+}$ tilt pattern (c) is similar as it possesses out-of-phase rotations about two directions with equal magnitude (perpendicular to the projection illustrated), but exhibits in-phase rotations $(+)$ about the remaining Cartesian direction with different amplitude (page normal). Periodic table adapted after Ref. 24

improper ferroelectricity, however, no comprehensive design prescription applicable to multiple chemistries has been proposed.

In this work, we present a materials design strategy to realize ferroelectricity in layered perovskite superlattices constructed from two different $A B \mathrm{O}_{3}$ materials, neither 
a

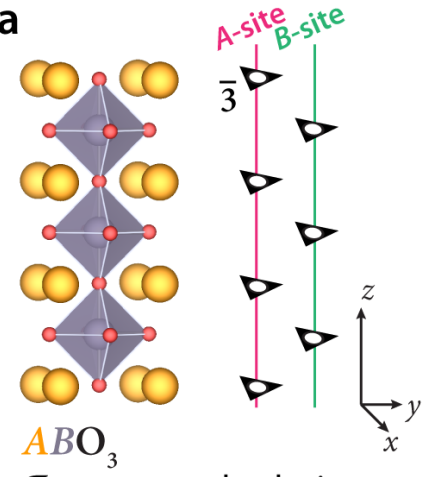

b

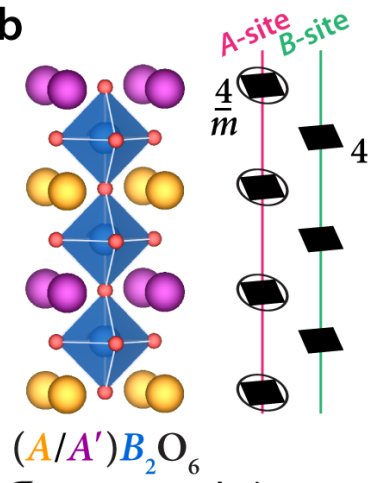

$\mathcal{I}$-centers on both sites

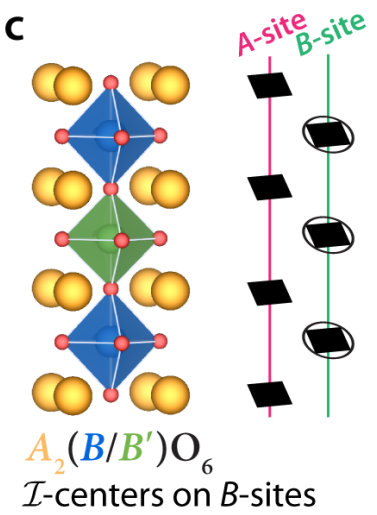

FIG. 2. Illustration of the Chemical Criterion for rotation-induced ferroelectricity in layered perovskite superlattices constructured from two different $A B \mathrm{O}_{3}$ perovskite materials. In (a) bulk $A B \mathrm{O}_{3}$ perovskites inversion $(\mathcal{I})$ centers are found on both the $A$ - and $B$-sites; the highest site-symmetry operator being a three-fold rotoinversion $(\overline{3})$. Cation ordering in layered perovskites, however, lifts the inversion centers on the $B$-site (leading to a 4 -fold rotation) in the $A / A^{\prime}$ layered perovskites (b) and on the $A$-site in the $B / B^{\prime}$ (c) structures. Inversion remains through the $\frac{4}{m}$ operation found on the remaining $A$-site and $B$-site, respectively. Since rotations of octahedra preserve the inversion on $B$-sites yet can remove it on the $A$-sites, only $A / A^{\prime}$ support this form of hybrid improper ferroelectricity.

of which are ferroelectric in bulk. The ferroelectricity is of a hybrid improper form,,$\sqrt{32} \sqrt{34}$ where the polarization $P$ can be induced by the coexistence of two octahedral rotation patterns of different symmetry. The design rules are constructed from group-theoretical methods combined with principles of perovskite crystal chemistry and are therefore completely general. In particular for $\left(A B \mathrm{O}_{3}\right)_{1} /\left(A^{\prime} B^{\prime} \mathrm{O}_{3}\right)_{1}$ superlattices, a layered $A / A^{\prime}$ cation ordering, designated as a chemical criterion, is required to realize this new form of ferroelectricity, whereas optimal materials selection, corresponding to an energetic criterion, is based solely on the rotation patterns present in the parent single phase $A B \mathrm{O}_{3}$ and $A^{\prime} B \mathrm{O}_{3}$ bulk perovskites. We find that the parent materials do not need to exhibit ferroelectricity, but rather that each constituent should have a tendency to distort in the orthorhombic Pnma $\left(a^{-} a^{-} c^{+}\right.$in Glazer notation, see Fig. 1. $)$ octahedral tilt pattern in bulk. Using ab initio first-principle techniques we test the design rules against artificially layered gallate and aluminate perovskites. (Note that our focus on $d^{0}$ cations is to avoid the known challenges of first-principles theory in treating materials with partially filled $d$ - and/or $f$-shells and is not a limitation of our strategy.) These materials design rules are capable of rapidly guiding the discovery of numerous unknown multifunctional materials as the $A / A^{\prime}$ cation layered ordering is highly amenable to advanced solid-state synthesis ${ }^{36 \mid 37}$ and layer-by-laye ${ }^{38}$ deposition techniques. Additionally, the targeted $a^{-} a^{-} c^{+}$ octahedral tilt pattern is the most common type observed in perovskites.

\section{Structure-property guidelines}

Hybrid improper ferroelectricity (HIF) has been shown to arise from a peculiar trilinear lattice coupling term 32134 in the thermodynamic free energy, $g P\left(Q_{1} Q_{2}\right)$, where the polarization $P$ is induced by the product of two translation symmetry-breaking lattice modes, $Q_{1}$ and $Q_{2}$, of different symmetry. The interaction strength of the coupled modes is described by the temperature-independent coefficient $g$. The principal design challenge is identifying the universal structural-chemical requirements within a class of materials that allows the non-polar lattice modes to induce ferroelectricity. Here, we focus on octahedral rotation-induced ferroelectricity in perovskite-structured oxides due to the abundance of $Q$ modes describing the $\mathrm{BO}_{6}$ connectivity.

Our crystal-chemistry design approach is to consider the archetypal five-atom cubic $A B \mathrm{O}_{3}$ perovskite as a basic chemical unit, interleaving any two perovskites to form a layered bi-color superlattice structure. [It is worth remembering that neither Glazer octahedral rotation patterns in bulk perovskites nor cation layering of the $A / A^{\prime}$ (or $\left.B / B^{\prime}\right)$ sites in such bi-color superlattices are capable of individually producing noncentrosymmetric structures.] We consider $\left(A B \mathrm{O}_{3}\right)_{1} /\left(A^{\prime} B \mathrm{O}_{3}\right)_{1}$ and $\left(A B \mathrm{O}_{3}\right)_{1} /\left(A B^{\prime} \mathrm{O}_{3}\right)_{1}$ superlattices, corresponding to the bulk-like compositions $\left(A, A^{\prime}\right) B_{2} \mathrm{O}_{6}$ and $A_{2}\left(B, B^{\prime}\right) \mathrm{O}_{6}$ (Sec. SI) with layered cation-orderings (Fig. 2), as the simplest structures that allow us to formulate materials selection rules in terms of the properties of the two bulk constituents: The chemical composition of the basic perovskite units considered, and the energetic or lattice dynamical properties of the five-atom perovskite units.

Chemical Criterion: The synthetic perovskites require an $A / A^{\prime}$ layered cation ordering for the octahedral rotations to induce ferroelectricity. The crystallographic rational for this criterion is as follows: In the $A B \mathrm{O}_{3}$ perovskite structure both the $A$-site and the $B$-site positions have local inversion symmetry (Fig. 2a). Rotations can lift the inversion center on the $A$-site, but always preserve the centricity (inversion on the $B$-site) of the octahedra. 


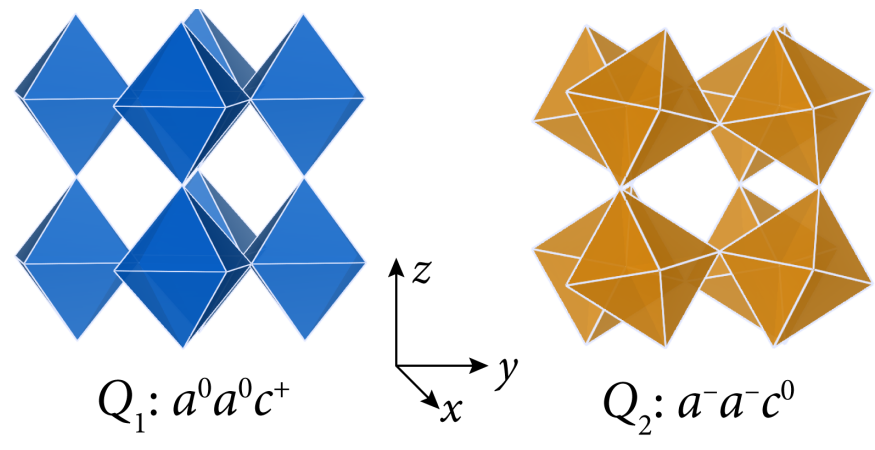

FIG. 3. The primary rotational modes $Q_{1}\left(a^{0} a^{0} c^{+}\right)$and $Q_{2}$ $\left(a^{-} a^{-} c^{0}\right)$ targeted to combine in the $A / A^{\prime}$ superlattices to induce hybrid improper ferroelectricity. The combination of these rotations produces the common orthorhombic $\left(a^{-} a^{-} c^{+}\right)$ tilt pattern found in perovskites.

$A / A^{\prime}\left(B / B^{\prime}\right)$ layered cation ordering removes the inversion center on the $B$-site ( $A$-site) as shown in Fig. $2 \mathrm{~b}$ and c. Therefore the combination of $A / A^{\prime}\left(B / B^{\prime}\right)$ cation order and rotations allows for (forbids) hybrid improper ferroelectricity.

This heuristic understanding is verified by our group theoretic methods. We find seven unique tilts patterns obtained from pairs of centric $B \mathrm{O}_{6}$ octahedral rotations patterns in the paraelectric $\left(A, A^{\prime}\right) B_{2} \mathrm{O}_{6}$ structure. Each combination leads to a $P\left(Q_{1} Q_{2}\right)$ term in the free energy (Table S5) allowing for rotation-induced ferroelectricityneither SOJT cations nor polar cation displacements are required. Note that the form of the trilinear term indicates that the combined rotation pattern is effectively polar; once the two rotations are present, there is only one direction for the induced polarization, i.e., turning on the two rotations is analogous to turning on an electric field. In contrast, we find no trilinear invariants in the paraelectric $A_{2}\left(B, B^{\prime}\right) \mathrm{O}_{6}$ structure and therefore hybrid improper ferroelectricity is forbidden.

The chemical criterion is insufficient to guarantee that the octahedral rotations induce a polarization, i.e., that $Q_{1} Q_{2} \rightarrow P$. Rather, the form of the trilinear coupling, $P\left(Q_{1} Q_{2}\right)$, compels only the mutual coexistence of such lattice distortions, which are commonly incompatible with one another, if any two are unstable 39

Energetic Criterion: The ground state structure of the $A B \mathrm{O}_{3}$ and the $A^{\prime} B \mathrm{O}_{3}$ building blocks must each contain the corresponding $Q_{1}$ and $Q_{2}$ rotation patterns. This requirement provides an optimal condition for hybrid improper ferroelectricity: it ensures the two octahedral rotations in the synthetic superlattice dominate the energy landscape over other competing instabilities, indicating they drive the transition to the polar structure. Said another way, the ground state structure of both bulk chemical units should possess the rotation pattern which results from the specific combination of octahedral rotations modes, $Q_{1}$ and $Q_{2}$, that are desired to be present in the $1 / 1 A B \mathrm{O}_{3} / A^{\prime} B \mathrm{O}_{3}$ superlattice.

This criterion stems from the fact that the rotation patterns are fully coherent in the synthetic $A / A^{\prime}$ structures. A rotational instability that appears in only one of the constituents, although likely capable of inducing the same rotation pattern in the second chemical unit, $\stackrel{40}{b}$ would produce a tilt pattern with overall smaller octahedral rotations and modulated rotation angle amplitudes. Such compound tilt patterns are detrimental to cooperative ordering of local dipoles and could hinder octahedral rotation-induced ferroelectricity.

\section{First-principles guided materials design}

Here we apply our criteria in order to select bulk single phase perovskite oxides with suitable chemical compositions and rotational patterns for integration into the $1 / 1$ ordered $A B \mathrm{O}_{3} / A^{\prime} B \mathrm{O}_{3}$ perovskites. The first design criterion is necessarily satisfied by restricting our investigation to this composition. Next we apply group-theoretic methods and consider all symmetry-adapted normal mode patterns (Table S5) that produce octahedral tilt patterns in the paraelectric $\left(A B \mathrm{O}_{3}\right)_{1} /\left(A^{\prime} B \mathrm{O}_{3}\right)_{1}$ structure. This analysis reveals many different possible invariants (Eq. $\mathrm{S} 1)$ that permit the octahedral rotations to induce ferroelectric polarizations through the trilinear $P Q_{1} Q_{2}$ term in the free energy. To narrow the search, we focus on the lattice modes $Q_{1}=a^{0} a^{0} c^{+}$and $Q_{2}=a^{-} a^{-} c^{0}$ (Fig. 3). These rotational modes are prime distortions to target because they are ubiquitous in single phase perovskites; they produce orthorhombic Pnma perovskites with the $a^{-} a^{-} c^{+}$octahedral tilt pattern.

We choose to focus on five different gallate and aluminate perovskites: $\mathrm{YAlO}_{3}, \mathrm{LaAlO}_{3}, \mathrm{BiAlO}_{3}, \mathrm{LaGaO}_{3}$, and $\mathrm{YGaO}_{3}$. We evaluate the lattice stability from calculations of the force constants throughout the Brillouin zone of these bulk compounds (Fig. S2) and perform (zero kelvin) full structural relaxations in various isotropy subgroups corresponding to the condensation of one or more of the unstable modes at the high-symmetry points within the Brillouin zone. In Table I we enumerate the most unstable phonon modes at the high symmetry points in the tetragonal Brillouin zone of the non-polar $(P 4 / \mathrm{mmm})$ bulk phases and identify the rotational pattern of the perovskite ground state. Note, although some of these bulk compounds show polar $\Gamma$-point instabilities, $\frac{41}{1}$ none of them exhibit ferroelectric perovskite ground state structures in the bulk except for $R 3 c \mathrm{BiAlO}_{3}$.

The combination of our targeted octahedral rotation modes, $Q_{1}=a^{0} a^{0} c^{+}(M$-point $)$ and $Q_{2}=a^{-} a^{-} c^{0}(A$ point), determine the ground state tilt patterns of three of the five compounds: $\mathrm{LaGaO}_{3}, \mathrm{YGaO}_{3}$ and $\mathrm{YAlO}_{3}$ all exhibit the orthorhombic $a^{-} a^{-} c^{+}$rotation pattern at room temperature (Fig. 1p). In contrast, $\mathrm{LaAlO}_{3}$ and $\mathrm{BiAlO}_{3}$ display the rhombohedral $a^{-} a^{-} a^{-}$tilt pattern.

We consider four representative $1 / 1$ superlattice test cases based on these bulk candidate perovskites (Table II):

(1) $\mathrm{YAlO}_{3} / \mathrm{YGaO}_{3}$. - Both $\mathrm{YAlO}_{3}$ and $\mathrm{YGaO}_{3}$ exhibit strong $a^{0} a^{0} c^{+}$and $a^{-} a^{-} c^{0}$ instabilities, i.e., the targeted orthorhombic rotation pattern, but the $B / B^{\prime}$ ordering does not satisfy the chemical crite- 
TABLE I. Phonon modes of the single phase paraelectric aluminates and gallates used in the selection of bulk perovskites to combine and realize rotation-induced ferroelectricity. Calculated frequency and distortion-types of the most unstable phonon modes for the reference $P 4 / \mathrm{mmm}$ bulk phases of the constituent superlattice materials. Imaginary frequencies indicate energy lowering instabilities. The high-symmetry $k$-points have the following wave vectors: $\Gamma=(0,0,0), M=\left(\frac{1}{2}, \frac{1}{2}, 0\right)$, $R=\left(0, \frac{1}{2}, \frac{1}{2}\right)$, and $A=\left(\frac{1}{2}, \frac{1}{2}, \frac{1}{2}\right)$.

\begin{tabular}{|c|c|c|c|}
\hline $\begin{array}{c}\text { Material } \\
\text { (bulk tilt system) }\end{array}$ & $\omega\left(\mathrm{cm}^{-1}\right)$ & $k$-point & distortion-type \\
\hline $\mathrm{LaAlO}_{3}$ & $46.9 i$ & $M$ & $a^{0} a^{0} c^{+}$ \\
\hline \multirow[t]{2}{*}{$\left(a^{-} a^{-} a^{-}\right)$} & $135 i$ & $A$ & $a^{0} a^{0} c^{-}$ \\
\hline & $108 i$ & $A$ & $a^{-} a^{-} c^{0}$ \\
\hline \multirow{6}{*}{$\begin{array}{c}\mathrm{BiAlO}_{3} \\
\left(a^{-} a^{-} a^{-}\right)\end{array}$} & $175 i$ & $\Gamma$ & $z$-polarization \\
\hline & $158 i$ & $\Gamma$ & $x y$-polarization \\
\hline & $234 i$ & $M$ & $a^{0} a^{0} c^{+}$ \\
\hline & $211 i$ & $R$ & $a^{+} a^{+} c^{0}$ \\
\hline & $247 i$ & $A$ & $a^{0} a^{0} c^{-}$ \\
\hline & $232 i$ & $A$ & $a^{-} a^{-} c^{0}$ \\
\hline \multirow{5}{*}{$\begin{array}{c}\mathrm{YAlO}_{3} \\
\left(a^{-} a^{-} c^{+}\right)\end{array}$} & $96 i$ & $\Gamma$ & $z$-polarization \\
\hline & $248 i$ & $M$ & $a^{0} a^{0} c^{+}$ \\
\hline & $229 i$ & $R$ & $a^{+} a^{+} c^{0}$ \\
\hline & $291 i$ & $A$ & $a^{0} a^{0} c^{-}$ \\
\hline & $282 i$ & $A$ & $a^{-} a^{-} c^{0}$ \\
\hline \multirow{4}{*}{$\begin{array}{c}\mathrm{LaGaO}_{3} \\
\left(a^{-} a^{-} c^{+}\right)\end{array}$} & $177 i$ & $M$ & $a^{0} a^{0} c^{+}$ \\
\hline & $165 i$ & $R$ & $a^{+} a^{+} c^{0}$ \\
\hline & $215 i$ & $A$ & $a^{0} a^{0} c^{-}$ \\
\hline & $209 i$ & $A$ & $a^{-} a^{-} c^{0}$ \\
\hline \multirow{6}{*}{$\begin{array}{c}\mathrm{YGaO}_{3} \\
\left(a^{-} a^{-} a^{+}\right.\end{array}$} & $180 i$ & $\Gamma$ & $z$-polarization \\
\hline & $144 i$ & $\Gamma$ & $x y$-polarization \\
\hline & $297 i$ & $M$ & $a^{0} a^{0} c^{+}$ \\
\hline & $286 i$ & $R$ & $a^{+} a^{+} c^{0}$ \\
\hline & $327 i$ & $A$ & $a^{0} a^{0} c^{-}$ \\
\hline & $322 i$ & $A$ & $a^{-} a^{-} c^{0}$ \\
\hline
\end{tabular}

${ }^{a}$ When confined to a perovskite manifold of structures.

rion, indicating that a trilinear coupling is symmetry forbidden.

(2) $\mathrm{LaGaO}_{3} / \mathrm{YGaO}_{3}$.- To mitigate this issue, we substitute $\mathrm{YAlO}_{3}$ with $\mathrm{LaGaO}_{3}$ to recover the trilinear coupling enabled by the chemical criterion. Since we find strong $a^{0} a^{0} c^{+}$and $a^{-} a^{-} c^{0}$ rotations present in both compounds (Table II), we anticipate that the energetic criterion will be strongly satisfiedrotation-driven ferroelectricity should be active in the $\mathrm{LaGaO}_{3} / \mathrm{YGaO}_{3}$ superlattice.

(3) $\mathrm{LaAlO}_{3} / \mathrm{YAlO}_{3}$.- - Starting again from (1), we will satisfy the chemical criterion by substitution of $\mathrm{YGaO}_{3}$ with $\mathrm{LaAlO}_{3}$. In contrast to case (2), the energetic requirement in this all-aluminate superlattice is weakly satisfied because $\mathrm{LaAlO}_{3}$ displays the rhombohedral tilt pattern in its ground state rather than the targeted orthorhombic one; this is reflected in the substantially harder $a^{0} a^{0} c^{+}$and $a^{-} a^{-} c^{0}$ mode frequencies.
(4) $\mathrm{LaAlO}_{3} / \mathrm{BiAlO}_{3}$.-Finally, rotation-induced ferroelectricity should be deactivated by substituting $\mathrm{YAlO}_{3}$ with $\mathrm{BiAlO}_{3}$ because neither compound possesses the orthorhombic Pnma tilt pattern - a direct violation of the energetic criterion despite satisfying the chemical restriction. Proper ferroelectricity could emerge, however, from the strong polar instability present in $\mathrm{BiAlO}_{3}$.

\section{Results}

We find that all superlattice compositions with $A / A^{\prime}$ cation ordering have polar crystal structures consisting of cation displacements and octahedral rotations (Fig. S3). The calculated ferroelectric polarizations shown in Table II are comparable to conventional ferroelectrics, e.g. the prototypical perovskite $\mathrm{BaTiO}_{3}$ exhibits a $\sim 33 \mu \mathrm{C} \mathrm{cm}^{-2}$ polarization in is ground state structure ${ }^{42}$ Consistent with our materials guidelines, the $B / B^{\prime}$ cation ordered superlattice is centrosymmetric. For the polar structures, however, the origin for ferroelectricity remains to be identified. The necessary condition to realize rotation-induced ferroelectricity is an interaction between different rotation distortions that produces a net energy gain to the polar ground state from their mutual coexistence. We determine this interaction by analyzing the energy surface around the paraelectric parent structure in terms of the unstable lattice modes that produce the octahedral rotations present in the ground state structures. We show from this analysis that complete control over hybrid improper ferroelectricity is gained through application of our simple design criteria.

(1) $\mathrm{YAlO}_{3} / \mathrm{YGaO}_{3}$. - The centrosymmetric ground state structure exhibits anti-parallel cation displacements and the centric $a^{-} a^{-} c^{-}$tilt pattern. Here, adjacent octahedra rotate out-of-phase in all directions and the $x y$ rotation angle magnitude is modulated from Ga-layer to Al-layer along the $z$-direction (Fig. S4). This octahedral motif results from a combination of the $a^{0} a^{0} c^{-}$ and $a^{-} a^{-} c^{0}$ unstable zone-boundary instabilities of the paraelectric superlattice. However, the combination of $a^{0} a^{0} c^{-}$(or the symmetry equivalent but energetically unique $a^{0} a^{0} c^{+}$rotation) with the $a^{-} a^{-} c^{0}$ mode in the $B / B^{\prime}$ superlattices produces only centrosymmetric structures (Fig. S5) and therefore prohibits the octahedral

TABLE II. Conditions for hybrid improper ferroelectricity (HIF) in $1 / 1$ perovskite superlattices. For the octahedral rotations to induce a ferroelectric polarization in the cation ordered superlattices both the chemical $\left(A / A^{\prime}\right.$ ordering) and energetic (orthorhombic $a^{-} a^{-} c^{+}$tilt pattern) criteria must be satisfied.

\begin{tabular}{llcccc}
\hline \hline & & $\begin{array}{c}A / A^{\prime} \\
\text { ordering? }\end{array}$ & $\begin{array}{c}a^{-} a^{-} c^{+} \\
\text {tilt pattern? }\end{array}$ & $\begin{array}{c}\text { polarization } \\
\left(\mu \mathrm{C} \mathrm{cm}{ }^{-2}\right)\end{array}$ & HIF? \\
\hline (1) & $\mathrm{YAlO}_{3} / \mathrm{YGaO}_{3}$ & no & no & 0 & no \\
(2) & $\mathrm{LaGaO}_{3} / \mathrm{YGaO}_{3}$ & yes & yes, strong & 11.8 & yes \\
(3) & $\mathrm{LaAlO}_{3} / \mathrm{YAlO}_{3}$ & yes & yes, weak & 9.94 & yes \\
(4) & $\mathrm{LaAlO}_{3} / \mathrm{BiAlO}_{3}$ & yes & no & 9.62 & no \\
\hline \hline
\end{tabular}



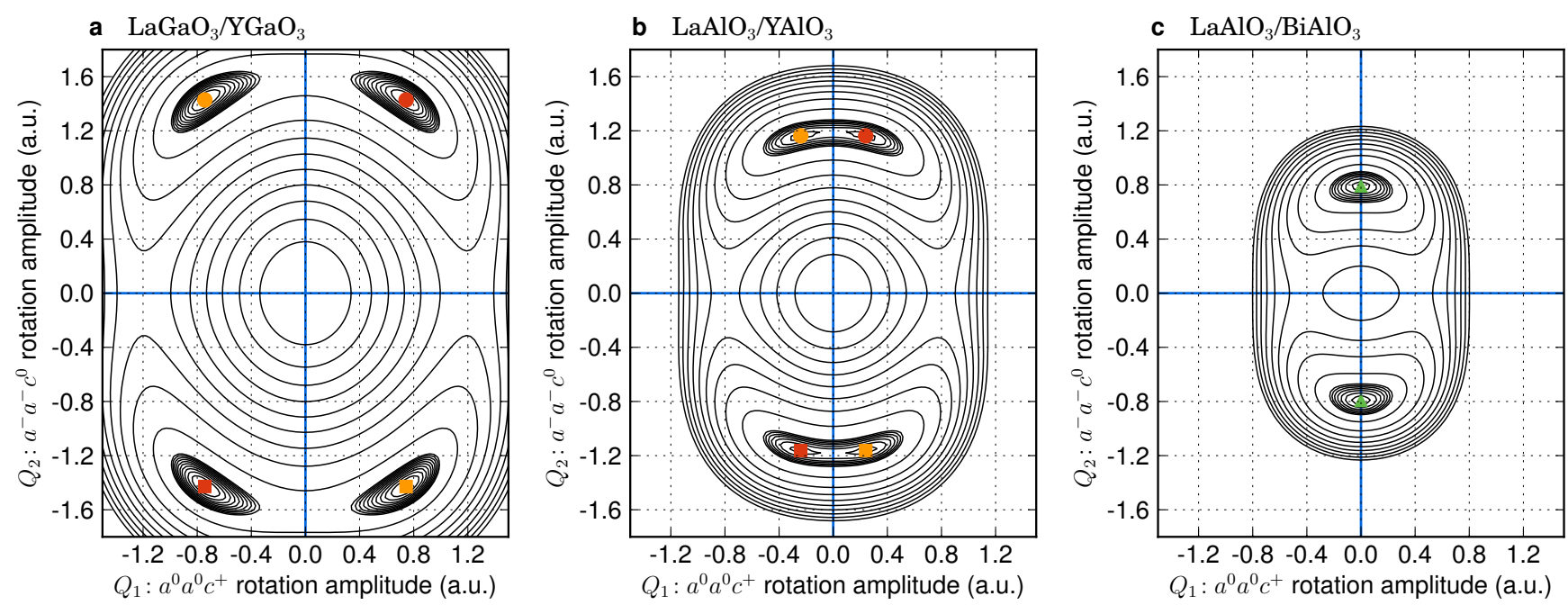

FIG. 4. Energetic signatures for rotation-induced ferroelectricity. The calculated zero kelvin two-dimensional energy surface contours for each cation-ordered perovskite with respect to the primary $Q_{1}: a^{0} a^{0} c^{+}$and $Q_{2}: a^{-} a^{-} c^{0}$ rotations centric octahedral rotation modes present in the polar ground state structures. Filled symbols indicate the positions of energy minima in the potential energy landscape. Equivalently colored symbols represent structures with identical ferroelectric polarization directions. Circles and squares differentiate anti-phase (domain) structures, which have identical polarization directions, but possess different non-polar structural distortion directions. In (a) and (b) the two $a^{0} a^{0} c^{+}$and $a^{-} a^{-} c^{0}$ rotations combine to produce the orthorhombic $a^{-} a^{-} c^{+}$tilt pattern: (a) The strong rotational modes in the $\mathrm{LaGaO}_{3} / \mathrm{YGaO}_{3}$ superlattice produce four polar crystal structures. Each $P m c 2_{1}$ structure is symmetry related to the others as either ferroelectric twin structures, which differ in

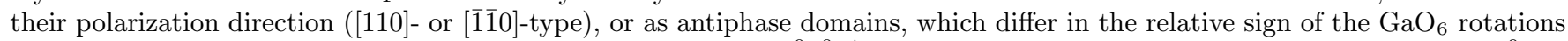
while the polarization direction remains fixed. (b) Despite the $a^{0} a^{0} c^{+}$rotation being substantially weaker than the $a^{-} a^{-} c^{0}$ tilt in the $\mathrm{LaAlO}_{3} / \mathrm{YAlO}_{3}$ structure, the combination of two non-polar octahedral rotations produce four symmetry related polar $P m c 2_{1}$ structures. The energy barrier separating the ferroelectric twins, however, is substantially reduced. (c) In the cation ordered $\mathrm{LaAlO}_{3} / \mathrm{BiAlO}_{3}$ superlattice this energy barrier collapses to zero: the $a^{0} a^{0} c^{+}$and $a^{-} a^{-} c^{0}$ strongly compete with each other to produce two symmetry equivalent non-polar (green triangles) Pmma structures, indicating the calculated ferroelectric polarization is not induced by the octahedral rotations.

rotations from inducing ferroelectricity. Consistent with this result, we find that these rotations also produce an identical non-polar structure in the Bi substituted analogue, $\mathrm{BiAlO}_{3} / \mathrm{BiGaO}_{3}$ (Fig. S6). Our first-principles predicted centrosymmetric $P 2_{1} / c$ structures confirm the symmetry-derived chemical criterion: Compositions with $B / B^{\prime}$ cation ordering prohibit the centric octahedra from producing a ferroelectric polarization.

(2) $\mathrm{LaGaO}_{3} / \mathrm{YGaO}_{3}$.- The ground state gallate superlattice exhibits the targeted orthorhombic $a^{-} a^{-} c^{+}$octahedral tilt pattern and possess polar, rather than fully compensated antiparallel, cation displacements. Adjacent $\mathrm{GaO}_{6}$ octahedra rotate coherently in-phase (with the same magnitude) about the axis perpendicular to the $\mathrm{La} / \mathrm{Y}$ ordering and out-of-phase in the two orthogonal directions (Fig. 3), which result from the combination of the highly unstable $a^{0} a^{0} c^{+}$and $a^{-} a^{-} c^{0}$ rotations present in the paraelectric $\mathrm{LaGaO}_{3}$ and $\mathrm{YGaO}_{3}$ phases (Table I) that are each independently energy lowering.

Rotation-induced ferroelectricity requires the two octahedral rotation modes present in the polar structure to dominate the energy landscape. To explore this, we map out the two-dimensional energy surface contours for the $\mathrm{LaGaO}_{3} / \mathrm{YGaO}_{3}$ superlattice in terms of these modes (Figure 4p): We find four symmetry equivalent energy min- ima (denoted by filled symbols) with noncentrosymmetric $P m c 2_{1}$ crystal structures, indicating that the $a^{0} a^{0} c^{+}$and $a^{-} a^{-} c^{0}$ rotations combine to produce the ferroelectric structure in the absence of cation displacements. If the two modes were strongly in competition with each other, producing an energy penalty by their mutual coexistence in the system, then only two minima would result. The location of these minima deep inside the quadrants and the large energy barrier $(\sim 39 \mathrm{meV} /$ f.u. $)$ separating the ferroelectric twins (at $Q_{1}>0$ and $<0$ ) reflects the strong favorable coupling between the largely unstable rotational modes possessed by both bulk constituents. This barrier is further enhanced by nearly $3 \mathrm{X}$ by substitution of $\mathrm{Y}$ with Bi (Fig. S7). For these reasons, we anticipate rotation-induced ferroelectricity in this composition to be robust to thermal effects at room temperature.

To verify that the $a^{-} a^{-} c^{+}$octahedral tilt pattern induces the electric polarization (and the subsequent cation displacements), we remove all polar distortions from the $P m c 2_{1}$ ground state structure and calculate the electroniconly contribution to the total polarization using the Berry phase method with only the octahedral rotations frozen in. $\stackrel{43}{ }$ Any electric polarization now must originate solely from the change in charge density induced by the cooperative and non polar oxygen displacements (octahedral 
rotations). Here, we find the rotations induce a sizable electronic-only polarization of $0.65 \mu \mathrm{C} \mathrm{cm}^{-2}$. The polarized charge density acts as an effective electric field, driving the ions to displace in a polar fashion - this is the essence of hybrid improper ferroelectricity. These induced cation displacements contribute to the total electric polarization in the fully relaxed $P m c 2_{1}$ structure. As a result, the polarization $P$ is intimately linked to the $a^{-} a^{-} c^{+}$tilt pattern in $\mathrm{LaGaO}_{3} / \mathrm{YGaO}_{3}$.

(3) $\mathrm{LaAlO}_{3} / \mathrm{YAlO}_{3}$. - The ground state polar structure of the all-aluminate composition is identical to the previous all-gallate superlattice: it exhibits the targeted $a^{-} a^{-} c^{+}$tilt pattern and polar cation displacements (space group $P m c 2_{1}$ ). As before, we find four symmetry equivalent energy minima resulting from the combination and cooperation of the $a^{0} a^{0} c^{+}$and $a^{-} a^{-} c^{0}$ rotations cooperate with one another (Fig. $4 \mathrm{~b}$ ). After full structural relaxation of this rotationally-only distorted structure (corresponding to the minima in Fig. 4b), we find polar cation displacements occurring in response to the $a^{-} a^{-} c^{+}$tilt pattern through the symmetry allowed trilinear $P_{z}\left(Q_{1} Q_{2}\right)$ coupling term absent in single phase non-polar perovskites.

Although the net interaction between the two rotation modes lower the energy of the $\mathrm{LaAlO}_{3} / \mathrm{YAlO}_{3}$ system, $Q_{2}$ is stronger than $Q_{1}$. This is evident from inspection of the unstable phonon modes for the bulk constituents (Table I) and is also discernible in Fig. $4 \mathrm{~b}$, where the energy minima are located close to the $Q_{1}=0$ boundary. This weak susceptibility to the $a^{0} a^{0} c^{+}$tilt system in paraelectric $\mathrm{LaAlO}_{3}$, leads to a small $(\sim 1 \mathrm{meV} /$ f.u. $)$ zero-kelvin energy barrier separating the two ferreoelectric twins. Thus the energetic balance between the two rotation modes and degree to which the energetic criterion is satisfied is equally as important as maintaining an $A / A^{\prime}$ cation ordered superlattice (chemical criterion). It determines the stability of octahedral rotation-induced HIF: Because the $a^{0} a^{0} c^{+}$rotation instability in $\mathrm{LaAlO}_{3}$ is weak, its presence in the ground state crystal structure is highly susceptible to finite temperature effects. If the four polar minima are thermally suppressed then two non-polar Pmma crystal structures would result, subverting the energetic criterion and nullifying the rotation-induced ferroelectricity mechanism.

(4) $\mathrm{LaAlO}_{3} / \mathrm{BiAlO}_{3}$. - The ground state polar structure exhibits the targeted $a^{-} a^{-} c^{+}$tilt pattern; however, Figure 4. illustrates that in the absence of polar ionic displacements, the energy interaction between the two $a^{0} a^{0} c^{+}$and $a^{-} a^{-} c^{0}$ rotations disfavors their mutual coexistence. Only two symmetry equivalent energy minima with centrosymmetric Pmma structures occur. In this extreme case, the energy cost of the coupling between rotations exceeds the gain from the individual modes. In other words, while the rotations $Q_{1}$ and $Q_{2}$ are of suitable symmetry to induce an electric polarization and are individually unstable (Table S4), our density functional calculations reveal that one mode dominates and suppresses the other. This happens in part due to the weak $Q_{1}$ instability in $\mathrm{LaAlO}_{3}$ and strong anharmonic coupling between $Q_{1}$ and $Q_{2}$ in bulk $\mathrm{BiAlO}_{3}$. Both of these features are reflected in the fact that neither lanthanum aluminate nor bismuth aluminate display the desired $a^{-} a^{-} c^{+}$tilt system in their ground states. The ferroelectric polarization that results in this system is due to a conventional soft polar mode; rotation-induced ferroelctricity is absent in the $\mathrm{LaAlO}_{3} / \mathrm{BiAlO}_{3}$ system.

\section{Outlook and summary}

Finally, we describe important applications exploiting the trilinear coupling that enables centric octahedral rotationinduced ferroelectricity. This mechanism supports electric polarizations in more diverse chemistries. Unlike the conventional SOJT-mechanism that lifts inversion symmetry and produces electric polarizations through cooperative displacements of cations with $d^{0}$ electronic configurations (group 4, 5 and 6 transition metal ions) or cations with stereochemical active $n s^{2}$ lone pair electrons, no such restriction is imposed on the cations' valence in this form of hybrid-improper ferroelectricity. Cations with strong magnetic interactions, open $d$ - or $f$-shell configurations, which are incompatible with conventional ferroelectricity, $\stackrel{44}{\text { are }}$ able to both fully coexist and couple to sizable electric polarizations: Centric octahedral rotation-induced ferroelectricity provides a plausible route to achieving robust magnetoelectric multiferroics.

It is interesting to conjecture that there are viable chemistries providing rotation-induced ferroelectrics with anomalously large electronic-only contributions to the total polarizations. In cases where the polarization contribution (and distortions) due to ionic displacements is also small, reversal of the electric polarization would require minimal ionic motion. These materials would satisfy many of the robust cycling and ultra fast (femtosecond timescale) dynamical switching requirements needed for next-generation electronics.

We have used first-principles density functional calculations combined with group theoretical studies to enumerate the crystal-chemistry design criteria required for the centric octahedral rotations pervasive in perovskite oxides to induce ferroelectric polarizations. We showed that interleaving two bulk perovskites to form an ordered and layered arrangement of $A / A^{\prime}$ cations [chemical composition $\left(A, A^{\prime}\right) B_{2} \mathrm{O}_{6}$ ] produces a new trilinear free energy term coupling three lattice modes: two octahedral rotations that dominate the energy landscape, forming the orthorhombic $a^{-} a^{-} c^{+}$ perovskite tilt system, and an electric polarization. For ordered perovskites where these conditions are satisfied, the trilinear term induces an electric polarization and hybrid improper ferroelectricity results. Because most octahedral rotations in perovskites freeze-in at elevated temperatures $(>300 \mathrm{~K})$, we argue that the trilinear coupling provides a robust route to realize synthetic ferroelectrics from constituents which are not ferroelectric in the bulk at room temperature. By leveraging systematic symmetry analysis with first-principles density functional 
calculations, we illustrated an a priori materials selection strategy for designing synthetic ferroelectric crystals only from knowledge of the lattice dynamics of the constituent materials.

\section{Methods}

Our zero kelvin density functional calculations are performed within the local density approximation (LDA) using the Vienna ab initio Simulation Package (VASP) and the projector augmented wave (PAW) method 47 with the following valence electron configurations: $5 s^{2} 5 p^{6} 5 d^{1} 6 s^{2}$ (La), $4 s^{2} 4 p^{6} 5 s^{2} 4 d^{1}$ (Y), $3 d^{10} 4 s^{2} 4 p^{1}$ (Ga), $3 s^{2} 3 p^{1}(\mathrm{Al})$ and $2 s^{2} 2 p^{4}(\mathrm{O})$. For the reference $P 4 / \mathrm{mmm}$ bulk perovskite structures we use a $5 \times 5 \times 5$ MonkhorstPack $k$-point mesh ${ }^{48}$ and a $500 \mathrm{eV}$ plane wave cutoff. We then volume-optimize each structure within $P 4 / \mathrm{mmm}$ symmetry. For our superlattice calculations we use a $5 \times 5 \times 3$ Monkhorst-Pack $k$-point mesh and a $550 \mathrm{eV}$ plane wave cutoff. We relax the ionic coordinates until the Hellmann-Feynman forces on the atoms are less than $0.1 \mathrm{meV}^{-1}$.

We obtain the phonon frequencies at high symmetry Brillouin zone points (Table I) by calculating total energies with respect to atomic displacements about the reference $P 4 / \mathrm{mmm}$ structure in a $2 \times 2 \times 2$ supercell. For these calculations we use a larger, $650 \mathrm{eV}$, planewave cutoff. In this frozen-phonon method, a series of small (symmetry inequivalent) atomic displacements are im- posed along different Cartesian directions. We calculate the dynamical matrix from the Hellman-Feynman forces and diagonalize the dynamical matrix to obtain the atomic displacement patterns (eigenvectors) and phonon mode frequencies (eigenvalues).

We obtain the ground state atomic structures for all cation ordered perovskite compositions by systematically "freezing-in" linear combinations of the unstable phonon modes (Table I) into the paraelectric cation-ordered perovskite reference structures (Sec. SII.B) and then performing full structural relaxations from first-principles.

\section{Acknowledgments}

The authors thank N.A. Benedek, E. Bousquet, A. Cano, K.R. Poeppelmeier, M. Stengel and K. Rabe for useful discussions and support from U.S. DOE, Office of Science, under Contract No. DE-AC02-06CH11357 (JMR) and the DOE-BES under Grant No. DE-SCOO02334 (CJF). Computational support was provided by the DOEBES, through the high-performance computing facilities at the Center for Nanoscale Materials and the LCRC operated FUSION compute cluster at Argonne National Laboratory. This work was initiated during a stay at the AQUIFER Program of the IMI and NSF under Award no. DMR-0843934, managed by the International Center for Materials Research, University of California, Santa Barbara, USA.
* jrondinelli@coe.drexel.edu

† fennie@cornell.edu

1 D. M. Newns, J. A. Misewich, C. C. Tsuei, A. Gupta, B. A. Scott, and A. Schrott, Applied Physics Letters 73, 780 (1998).

2 O. Auciello, J. F. Scott, and R. Ramesh, Physics Today 51, 22 (1998).

3 C. H. Ahn, J.-M. Triscone, and J. Mannhart, Nature 424, 1015 (2003).

${ }^{4}$ M. Rini, R. Tobey, N. Dean, J. Itatani, Y. Tomioka, Y. Tokura, R. W. Schoenlein, and A. Cavalleri, Nature 449, 72 (2007).

5 J. F. Scott, Ferroelectric memories (Springer-Verlag, 2000).

6 S. Hormoz and S. Ramanathan, Solid-State Electron. 54, 654 (2010).

7 H. Takagi and H. Hwang, Science 327, 1601 (2010).

8 J. Mannhart and D. Schlom, Science 327, 1607 (2010).

9 J. K. Burdett, Inorganic Chemistry 20, 1959 (1981).

10 M. Kunz and I. D. Brown, Journal of Solid State Chemistry 115, 395 (1995).

11 I. B. Bersuker, Chem. Rev. 101, 1067 (2001).

12 N. W. Thomas, Acta Cryst. B 52, 16 (1996).

13 M. Ghita, M. Fornari, D. J. Singh, and S. V. Halilov, Physical Review B 72, 054114 (2005).

14 J. B. Torrance, P. Lacorre, A. I. Nazzal, E. J. Ansaldo, and C. Niedermayer, Physical Review B 45, 8209 (1992).

15 H. W. Eng, P. W. Barnes, B. M. Auer, and P. M. Woodward, Journal of Solid State Chemistry 175, 94 (2003).

16 M. A. Subramanian, A. P. Ramirez, and W. J. Marshall,
Physical Review Letters 82, 1558 (1999).

17 J.-S. Zhou and J. B. Goodenough, Physical Review Letters 96, 247202 (2006).

18 A. J. Millis, Nature 392, 147 (1998).

19 T. Goto, T. Kimura, G. Lawes, A. P. Ramirez, and Y. Tokura, Physical Review Letters 92, 257201 (2004).

20 M. Imada, A. Fujimori, and Y. Tokura, Reviews of Modern Physics 70, 1039 (1998).

21 A. M. Glazer, Acta Cryst. B 28, 3384 (1972).

22 P. M. Woodward, Acta Cryst. B 53, 32 (1997).

23 P. M. Woodward, Acta Cryst. B 53, 44 (1997).

24 D. G. Schlom, C. D. Theis, and M. E. Hawley, in Integrated Thin Films and Applications, Vol. 86, edited by R. K. Pandey, D. E. Witter, and U. Varshney (American Ceramic Society, Westerville, 1998) pp. 41-60.

${ }^{25}$ H. T. Stokes, E. H. Kisi, D. M. Hatch, and C. J. Howard, Acta Cryst. B 58, 934 (2002).

26 P. A. Maggard, C. L. Stern, and K. R. Poeppelmeier, Journal of the American Chemical Society 123, 7742 (2001).

27 M. E. Welk, A. J. Norquist, F. P. Arnold, C. L. Stern, and K. R. Poeppelmeier, Inorganic Chemistry 41, 5119 (2002).

${ }^{28}$ M. R. Marvel, J. Lesage, J. Baek, P. S. Halasyamani, C. L. Stern, and K. R. Poeppelmeier, Journal of the American Chemical Society 129, 13963 (2007).

29 K. R. Heier, A. J. Norquist, C. G. Wilson, C. L. Stern, and K. R. Poeppelmeier, Inorganic Chemistry 37, 76 (1998).

30 P. A. Maggard, T. S. Nault, C. L. Stern, and K. R. Poeppelmeier, Journal of Solid State Chemistry 175, 27 (2003).

31 M. Yang, J. Orò-Solè, J. A. Rodgers, A. B. Jorge, A. Fuertes, 
and J. P. Attfield, Nat. Chem. 3, 47 (2011).

32 E. Bousquet, M. Dawber, N. Stucki, C. Lichtensteiger, P. Hermet, S. Gariglio, J.-M. Triscone, and P. Ghosez, Nature 452, 732 (2008).

33 I. Etxebarria, J. M. Perez-Mato, and P. Boullay, Ferroelectrics 401, 17 (2010).

34 N. A. Benedek and C. J. Fennie, Physical Review Letters 106, 107204 (2011).

35 T. Fukushima, A. Stroppa, S. Picozzi, and J. M. PerezMato, Phys. Chem. Chem. Phys. 13, 12186 (2011).

${ }^{36}$ G. King and P. M. Woodward, Journal of Materials Chemistry 20, 5785 (2010).

37 W. Dachraoui, T. Yang, C. Liu, G. King, J. Hadermann, G. Van Tendeloo, A. Llobet, and M. Greenblatt, Chemistry of Materials 23, 2398 (2011).

${ }^{38}$ P. Zubko, S. Gariglio, M. Gabay, P. Ghosez, and J.-M. Triscone, Annual Review of Condensed Matter Physics 2, 141 (2011).

39 In many cases, $1 / 1$ superlattices that satisfy this condition can be constructed from basic perovskite chemical units that are ferroelectric in the bulk, e.g. $\mathrm{PbTiO}_{3}: P$ then describes a conventional (soft transverse optical mode) ferroelectric instability thats coexists with the octahedral rotations, rather than emerging from them. It is therefore equally possible that the polarization and a single octahe- dral rotation distortion are the primary instabilities that break inversion symmetry and induce a second rotational distortion, $\stackrel{49}{ }$ i.e., $P Q_{2} \rightarrow Q_{1}$ (or $P Q_{1} \rightarrow Q_{2}$ ); this does not yield octahedral rotation-induced HIF.

40 J. M. Rondinelli and N. A. Spaldin, Physical Review B 82, 113402 (2010).

41 Although complicating the analysis, if a bulk material is a proper ferroelectric, the presence of a soft polar mode does not preclude the possibility of octahedral rotation-induced ferroelectricity.

42 W. Zhong, D. Vanderbilt, and K. M. Rabe, Physical Review Letters 73, 1861 (1994).

43 R. D. King-Smith and D. Vanderbilt, Physical Review B 47, R1651 (1993).

44 N. A. Hill, Journal of Physical Chemistry B 104, 6694 (2000).

45 G. Kresse and J. Furthmüller, Physical Review B 54, 11169 (1996).

${ }^{46}$ G. Kresse and D. Joubert, Physical Review B 59, 1758 (1999).

47 P. E. Blöchl, Physical Review B 50, 17953 (1994).

48 H. J. Monkhorst and J. D. Pack, Physical Review B 13 , 5188 (1976).

49 J. M. Perez-Mato, M. Aroyo, A. García, P. Blaha, K. Schwarz, J. Schweifer, and K. Parlinski, Physical Review B 70, 214111 (2004). 